\title{
Registros de dieta de opiliões na Mata Atlântica (Arachnida, Opiliones)
}

\author{
Luis Gustavo Talarico Rubim \\ Thiago Henrique dos Reis Pádua \\ Adriele Garcia Costa * \\ Marcos Magalhães de Souza \\ Instituto Federal de Educação, Ciência e Tecnologia do Sul de Minas Gerais \\ IFSULDEMINAS, Campus Inconfidentes, CEP 37.576-000, Inconfidentes - MG, Brasil \\ * Autor para correspondência \\ costaadriele50@gmail.com
}

Submetido em 31/03/2021

Aceito para publicação em 05/08/2021

\section{Resumo}

Diferentes estudos sugerem que os Opiliones apresentam hábitos alimentares generalistas. Entretanto, considerando a diversidade de opiliões no Brasil, o conhecimento da dieta desse grupo é limitado. Neste estudo, apresentamos 10 registros de alimentação praticados por três espécies e quatro morfoespécies, com primeiras informações para Gonyleptes atrus. Conduzimos o estudo em áreas de Mata Atlântica e urbanas no sul do estado de Minas Gerais, sudeste do Brasil, no período de dezembro de 2019 a março de 2020, totalizando 20 horas de esforço amostral. Nossos dados corroboram a dieta generalista dos opiliões, mas mais estudos podem contribuir para o conhecimento das especificidades de diferentes espécies.

Palavras-chave: Alimentação; Dieta; Etologia; Oportunista

\section{Abstract}

Report of harvestmen's diet (Arachnida, Opiliones). The literature suggests that Opiliones present generalist feeding habits. However, little is known about the diet of most species in Brazil. Here we present 10 feeding records observed in 3 species and 4 morphospecies, with one being the first register for Gonyleptes atrus. We conducted this study in Atlantic forest and urban areas from the southern Minas Gerais state, Brazil, from December 2019 to March 2020, based on a sampling effort of 20 hours. practiced. Our data corroborate the generalist feeding habit of the Opiliones order, but more studies are needed to evaluates differences between species.

Key words: Diet; Ethology; Feeding; Opportunist 
A dieta dos Opiliones (Arachnida) inclui invertebrados, como Annelida (HALAJ; CADY, 2000); Gastropoda (NYFFELER; SYMONDSON, 2001); Coleoptera (HALAJ; CADY, 2000), larvas de Lepidoptera (ASHBY; POTTINGER, 1974); Diptera (HVAM; TOFT, 2008) e Hemiptera (ALLARD; YEARGAN, 2005), pequenos vertebrados, como anfíbios (CASTANHO; PINTO-DA-ROCHA, 2005) e filhotes de aves (BENSON; CHARTIER, 2010); matéria em decomposição (CURTIS; MACHADO, 2007); e frutos (SCHAUS et al., 2013).

Tipicamente os opiliões repousam em locais úmidos durante o dia e forrageiam durante a noite (EDGAR, 1990). Como estratégias de forrageio, opiliões podem ser predadores de emboscada ou praticar busca ativa (ACOSTA et al.,1995; MACHADO et al., 2000), havendo inclusive relato de usurpação de presas (RAMIN, 2014). A estratégia de forrageio pode variar dependendo da preferência alimentar da espécie, sendo a de emboscada eficiente na captura de presa viva enquanto a de busca ativa é mais comum nas espécies com preferência alimentar por animais mortos e matéria vegetal (ACOSTA; MACHADO, 2007).

O Brasil possui a maior riqueza de opiliofauna do mundo (KURY, 2017), porém as informações sobre o hábito alimentar desses aracnídeos ainda são restritas a poucas espécies e a grupos específicos (ACOSTA;
MACHADO 2007; CHELINI; MACHADO, 2014). Neste sentido, o presente estudo objetiva ampliar as informações quanto às espécies animais que compõem a dieta dos opiliões.

Os registros ocorreram no período de dezembro de 2019 a março de 2020, com 10 dias de busca ativa das $18 \mathrm{~h}$ às $20 \mathrm{~h}$, totalizando $20 \mathrm{~h}$ de esforço amostral. As coletas foram realizadas em diferentes localidades no sul do estado de Minas Gerais, sudeste do Brasil: em Floresta Ombrófila Mista, no município de

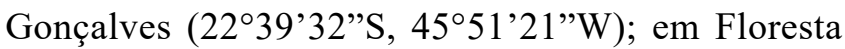
Estacional Semidecidual, no município de Inconfidentes (22 ${ }^{\circ} 19^{\prime} 01$ 'S, 46 ${ }^{\circ} 19^{\prime} 40^{\prime}$ 'W, ambas fitofisionomias de domínio da Mata Atlântica - OLIVEIRA-FILHO, 2006); em áreas urbanas no município de Ouro Fino (22 $\left.{ }^{\circ} 16^{\prime} 59^{\prime} \mathrm{S}, 46^{\circ} 22^{\prime} 08^{\prime \prime} \mathrm{W}\right)$. Os opiliões foram incorporados na coleção CBVS, sistema speciesLink, laboratório de zoologia do IFSULDEMINAS, Campus Inconfidentes, número de tombo 06217-2019 a 062262019, e as presas depositadas em via úmida sem número de tombo. O trabalho foi autorizado pelo IEF-MG 079/2019 e SISBIO 71076.

Registramos 10 eventos de alimentação praticados por três espécies e quatro morfoespécies, incluindo uma fase imatura de opiliões (Tabela 1). Há dados inéditos para dieta de Gonyleptes atrus e novas informações para a subfamília Gagrellinae.

TABELA 1: Registro de eventos de alimentação em Opiliones por táxon e localidade.

\begin{tabular}{|c|c|c|c|c|}
\hline Família & Espécie/ morfotipo & $\begin{array}{l}\text { Táxon do animal utilizado } \\
\text { como alimento }\end{array}$ & Macro-habitat & Micro-habitat \\
\hline \multicolumn{5}{|l|}{ Gonyleptidae } \\
\hline & $\begin{array}{l}\text { Acutisoma longipes } \\
\text { Roewer, } 1913\end{array}$ & $\begin{array}{l}\text { Formicinae sp., Lepidoptera } \\
\text { Pantherodes pardalaria } \\
\text { (Hübner, 1823) }\end{array}$ & Floresta ombrófila mista & $\begin{array}{l}\text { Vegetação } \\
\text { rasteira }\end{array}$ \\
\hline & Gonyleptidae gen. sp.1 & Tipulidae sp. (Diptera) & Floresta ombrófila mista & Barranco \\
\hline & Gonyleptidae gen. sp.2 & Fragmentos de Hymenoptera & Floresta ombrófila mista & $\begin{array}{l}\text { Vegetação } \\
\text { rasteira }\end{array}$ \\
\hline & $\begin{array}{l}\text { Gonyleptes atrus Mello- } \\
\text { Leitão, } 1923\end{array}$ & Oligochaeta, Urobenus sp. & Floresta ombrófila mista & Barranco \\
\hline & $\begin{array}{l}\text { Mischonyx cuspidatus } \\
\text { (Roewer, 1913) }\end{array}$ & $\begin{array}{l}\text { Formicidae sp.; Crustacea } \\
\text { (Isopoda sp.) }\end{array}$ & $\begin{array}{l}\text { Floresta Estacional } \\
\text { Semidecidual } \\
\text { Área urbana }\end{array}$ & Barranco \\
\hline & Pachylinae gen. sp.1 & Fragmentos de Diptera & Floresta ombrófila mista & Serrapilheira \\
\hline \multicolumn{5}{|c|}{ Sclerosomatidae } \\
\hline & Gagrellinae gen. sp.1 & Apidae, Trigona sp. & Floresta ombrófila mista & Barranco \\
\hline & Gagrellinae gen. sp.2 & Oligochaeta sp. & Floresta ombrófila mista & Serrapilheira \\
\hline
\end{tabular}


A dieta dos opiliões se mostrou variada (Tabela 1), sendo constituída por insetos das ordens Hymenoptera (Figura 1), Diptera e Lepidoptera (70\% das presas), mas também por crustáceos e minhocas.

FIGURA 1: Gagrellinae gen. sp. 1 se alimentando de Trigona sp. (Apidae), registro em área de Floresta Mista, sul do estado de Minas Gerais.

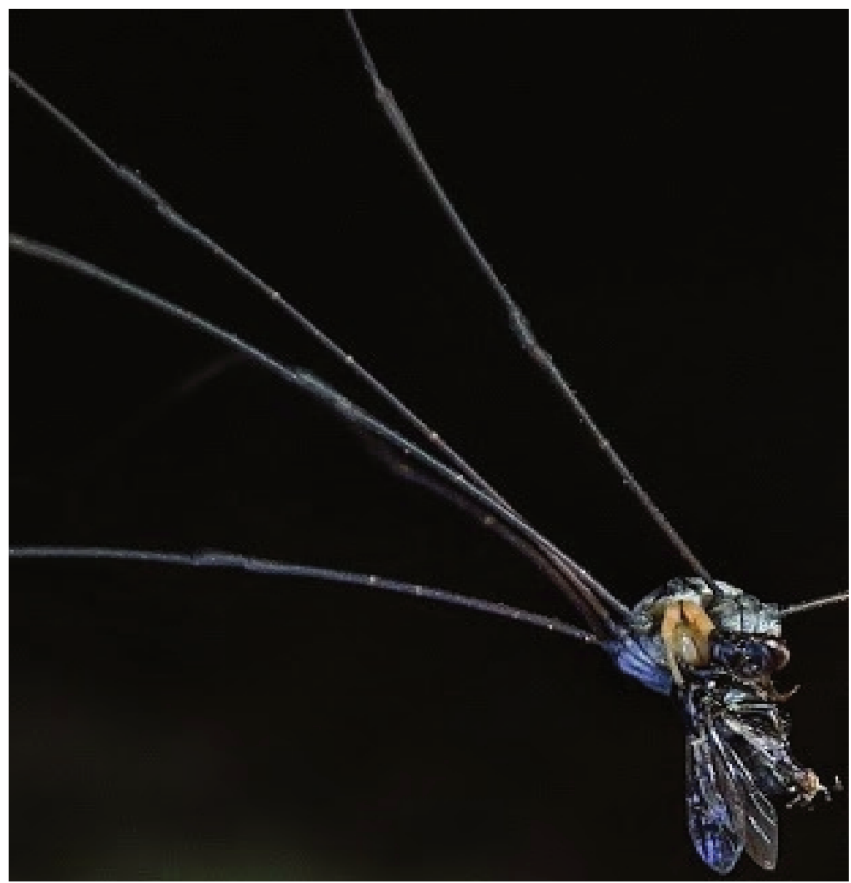

Embora seja sugerido que os opiliões são onívoros generalistas, há alguns táxons paleárticos que são especialistas em predar gastrópodes (NYFFELER; SYMONDSON, 2001). Entretanto, pouco se conhece sobre a dieta da maioria das espécies (ACOSTA; MACHADO, 2007), pois muitos dos relatos são provenientes de observações episódicas.

Neste estudo apresentamos o primeiro registro da dieta de G. atrus (Tabela 1). Importante destacar que não observamos o ato de predação em si, mas o item presente no aparelho bucal dos opiliões. Portanto, a presa pode eventualmente ter sido coletada morta, já que o hábito necrófago é comum em opiliões (HALAJ; CADY, 2000). Para a subfamília gagrellinae há registro do gênero Jussara se alimentando de ninfas de Neosadocus maximus (Gonyleptidae), outra espécie de opilião, comportamento também relatado para outras espécies (CHELINI; MACHADO, 2014).
Registramos Mischonyx cuspidatus se alimentando de Isopoda, hábito já reportado para outras espécies (WILLEMART et al., 2007). Esses crustáceos são comuns em ambientes urbanos (KENNE; ARAUJO, 2015), procurando refúgios embaixo de tijolos e de outros substratos, condição em que também se encontra M. cuspidatus, espécie sinantrópica e de ampla distribuição geográfica (COSTA et al., 2020). Também observamos $M$. cuspidatus se alimentando de formigas, e há registros de outros animais em sua dieta (PEREIRA et al., 2004), evidenciando uma dieta generalista nessa espécie de aracnídeo, de acordo com o que vem sendo sugerido como o hábito alimentar mais comum em Opiliones.

A realização deste estudo ampliou informações quanto às espécies animais que compõem a dieta dos opiliões. Contudo, as informações sobre o hábito alimentar desses aracnídeos ainda são restritas a poucas espécies. Portanto se faz necessária a realização de novos trabalhos para diminuir essa lacuna do conhecimento, sobretudo em áreas de Cerrado e Caatinga, biomas pouco conhecidos quanto à opiliofauna (DeSOUZA et al., 2017), e que estão sofrendo rápida redução de sua área natural.

\section{Agradecimentos}

Ao Dr. Ludson Neves de Ázara pela identificação dos opiliões, e aos pesquisadores: Dr. Jamil Moraes (IFSULDEMINAS, Campus Inconfidentes), Dra. Juliane Floriano Lopes Santos (UFJF), doutorando Marco Antônio de Menezes Ferreira (UFRJ) pelas identificações das presas. Françueudo Bezerra da Silva pelas imagens em campo e em laboratório. Ao SISBIO e IEF-MG pela concessão das licenças. A Gláucia Stefani Santos, por toda ajuda em campo e logística, e ao Instituto Federal de Educação, Ciência e Tecnologia do Sul de Minas Gerais e ao Dr. Ricardo Pinto da Rocha (USP) pelo auxílio no texto.

\section{Referências}

ACOSTA, L. E.; MACHADO, G. Diet and foraging. In:PINTO-DAROCHA, R.; MACHADO, G.; GIRIBET, G. (Ed.). Harvestmen: 
the biology of opiliones. Cambridge: Harvard University Press, 2007. p. 309-338.

ACOSTA, L. E.; PEREYRA, F. E.; PIZZI, R. A. Field observation on Pachyloidellus goliath (Opiliones, Gonyleptidae) in Pampa de Achala, province of Córdoba. Bulletin of the British Arachnological Society, London, v. 10, n. 1 p. 23-28, 1995.

ALLARD, C. M.; YEARGAN, K. V. Effect of diet on development and reproduction of the harvestman Phalangium opilio (Opiliones: Phalangiidae). Environmental Entomology, Lanham, v. 34, n. 1, p. 6-13, 2005.

ASHBY, J. W.; POTTINGER, R. P. Natural regulation of Pieris rapae Linnaeus (Lepidoptera: Pieridae) in Canterbury, New Zealand. New Zealand Journal of Agricultural Research, Lincoln, v. 17, n. 2, p. 229-239, 1974.

BENSON, T. J.; CHARTIER, N. A. Harvestmen as predators of bird nestlings. Journal of Arachnology, Chicago, v. 38, n. 2, p. 374-376, 2010.

CASTANHO, L. M.; PINTO-DA-ROCHA, R. Harvestmen (Opiliones: Gonyleptidae) predating on treefrogs (Anura: Hylidae). Revista de Aracnologia, Zaragoza, v. 11, n. 1, p. 43-45, 2005.

CHELINI, M. C.; MACHADO, G. Multiple lines of egg defense in a Neotropical Arachnid with temporary brood desertion. Ethology, Weinheim, v. 120, n. 12, p. 1147-1154, 2014.

COSTA, A. G.; ÁZARA, L. N.; CLEMENTE, M. A.; SOUZA, M. M. Opiliofauna (Arachnida, Opiliones) of the Seasonal Semidecidual Forest of the State of Minas Gerais, Brazil. Biotemas, Florianópolis, v. 33, n. 3, p. 1-11, 2020.

CURTIS, D. J.; MACHADO, G. Ecology. In: PINTO-DA-ROCHA, R.; MACHADO, G.; GIRIBET, G. (Ed.). Harvestmen: the biology of Opiliones. Cambridge: Harvard University Press, 2007. p. 280308.

DeSOUZA, A. M.; DaSILVA, M. B.; CARVALHO, L. S. Opiliões Laniatores do semiárido: grandes achados taxonômicos com o pouco que se conhece. In: Freddy Bravo. (Org.). Artrópodes do Semiárido II: Biodiversidade e conservação. 2. ed. São Paulo: Métis Produção Editorial, v. 139, p. 7-27, 2017.

EDGAR, A. L. Opiliones (Phalangida). In: DANIEL. L. D. (Ed.). Soil Biology guide. New York: John Wiley \& Sons, 1990. p. 529581.

HALAJ, J.; CADY, A. B. Diet composition and significance of earthworms as food of harvestmen (Arachnida: Opiliones). The
American Midland Naturalist, South Bend, v. 143, n. 2, p. 487491, 2000.

HVAM, A.; TOFT. S. Prey preference and consumption by some non-specialist harvestman species (Arachnida: Opiliones). Arachnology, London, v. 14, n. 4, p. 198-205, 2008.

KENNE, D. C.; ARAUJO, P. B. Balloniscus glaber (Crustacea, Isopoda, Balloniscidae), a habitat specialist species in a disturbed area of Brazil. Iheringia, Série Zoologia, Porto Alegre, v. 105, n. 4, p. 430-438, 2015.

KURY, A. B. Classificação dos Opiliones. Rio de Janeiro: Museu Nacional/UFRJ, 2017. Disponível em <http:/www.museunacional. ufrj.br/mndi/Aracnologia/opiliones.html>.

MACHADO, G.; RAIMUNDO, R. L. G.; OLIVEIRA, P. S. Daily activity schedule, gregariousness, and defensive behaviour in the neotropical harvestman Goniosoma longipes (Opiliones: Gonyleptidae). Journal of Natural History, London, v. 34, n. 4, p. 587-596, 2000.

NYFFELER, M. W.; SYMONDSON. O. C. Spiders and harvestmen as gastropod predators. Ecological Entomology, London, v. 26, n. 6, p. 617-628, 2001.

OLIVEIRA-FILHO, A. T. Definição e delimitação de domínios e subdomínios das paisagens naturais do estado de Minas Gerais. In: SCOLFORO, J. R.; CARVALHO, L. M. T. (Ed.). Mapeamento e inventário da flora e dos reflorestamentos de Minas Gerais. Lavras: Universidade Federal de Lavras, 2006. p. 21-35.

PEREIRA, W.; ELPINO-CAMPOS, A.; DEL-CLARO, K.; MACHADO, G. Behavioral repertory of the neotropical harvestman Ilhaia cuspidata (Opiliones, Gonyleptidae). The Journal of Arachnology, Chicago, v. 32, n. 1, p. 22-30, 2004.

RAMIN, A. Z. Deslocamento ao longo da noite e outros aspectos da biologia do opilião Serracutisoma pseudovarium no Parque Estadual Intervales, São Paulo, Brasil. 2014. 107 f. Dissertação (Mestrado) - Universidade de São Paulo, São Paulo. 2014.

SCHAUS, M. H.; TOWNSEND, V. R.; ILLINIK, J. J. Food choice of the neotropical harvestman Erginulus clavotibialis (Opiliones: Laniatores: Cosmetidae). Journal of Arachnology, Chicago, v. 4, n. 2, p. 219-221, 2013.

WILLEMART, R. H.; CHELINI, M. C.; DE ANDRADE, R.; GNASPINI, P. An ethological approach to a SEM survey on sensory structures and tegumental gland openings of two neotropical harvestmen (Arachnida, Opiliones, Gonyleptidae). Italian Journal of Zoology, Modena, v. 74, n. 1, p. 39-54, 2007. 